\title{
Excessive aggregation of membrane proteins in the Martini model
}

\author{
Matti Javanainen ${ }^{1,2}$, Hector Martinez-Seara ${ }^{1,3}$, Ilpo Vattulainen ${ }^{1,2,4 *}$ \\ 1 Laboratory of Physics, Tampere University of Technology, Tampere, Finland, 2 Department of Physics, \\ University of Helsinki, Helsinki, Finland, 3 Institute of Organic Chemistry and Biochemistry, Czech Academy \\ of Sciences, Prague, Czech Republic, 4 Memphys - Center for Biomembrane Physics \\ * hseara@gmail.com (HMS); ilpo.vattulainen@ helsinki.fi (IV)
}

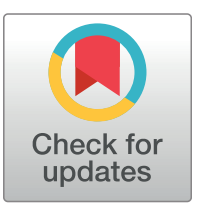

\section{OPEN ACCESS}

Citation: Javanainen M, Martinez-Seara $\mathrm{H}$, Vattulainen I (2017) Excessive aggregation of membrane proteins in the Martini model. PLOS ONE 12(11): e0187936. https://doi.org/10.1371/ journal.pone.0187936

Editor: Elena Papaleo, Danish Cancer Society Research Center, DENMARK

Received: August 15, 2017

Accepted: October 27, 2017

Published: November 13, 2017

Copyright: @ 2017 Javanainen et al. This is an open access article distributed under the terms of the Creative Commons Attribution License, which permits unrestricted use, distribution, and reproduction in any medium, provided the original author and source are credited.

Data Availability Statement: All data discussed in this work are openly available from www.zenodo. org under Creative Commons Attribution 4.0 license: Umbrella Sampling Simulations of TM Domain Dimerization (DOI:10.5281/zenodo. 1019733); Simulations of Spontaneous TM Domain Dimer Formation (DOI:10.5281/zenodo. 1009183).

Funding: MJ, HMS, and IV thank the Academy of Finland (Centre of Excellence program (grant no. 307415)) and the European Research Council (Advanced Grant CROWDED-PRO-LIPIDS (grant

\section{Abstract}

The coarse-grained Martini model is employed extensively to study membrane protein oligomerization. While this approach is exceptionally promising given its computational efficiency, it is alarming that a significant fraction of these studies demonstrate unrealistic protein clusters, whose formation is essentially an irreversible process. This suggests that the protein-protein interactions are exaggerated in the Martini model. If this held true, then it would limit the applicability of Martini to study multi-protein complexes, as the rapidly clustering proteins would not be able to properly sample the correct dimerization conformations. In this work we first demonstrate the excessive protein aggregation by comparing the dimerization free energies of helical transmembrane peptides obtained with the Martini model to those determined from FRET experiments. Second, we show that the predictions provided by the Martini model for the structures of transmembrane domain dimers are in poor agreement with the corresponding structures resolved using NMR. Next, we demonstrate that the first issue can be overcome by slightly scaling down the Martini protein-protein interactions in a manner, which does not interfere with the other Martini interaction parameters. By preventing excessive, irreversible, and non-selective aggregation of membrane proteins, this approach renders the consideration of lateral dynamics and protein-lipid interactions in crowded membranes by the Martini model more realistic. However, this adjusted model does not lead to an improvement in the predicted dimer structures. This implicates that the poor agreement between the Martini model and NMR structures cannot be cured by simply uniformly reducing the interactions between all protein beads. Instead, a careful amino-acid specific adjustment of the protein-protein interactions is likely required.

\section{Introduction}

Aggregation of proteins has severe implications for health. For instance, $G$ protein-coupled receptors (GPCRs) form complex functional oligomers that act as drug targets in membranes $[1,2]$. Also, the aggregation of misfolded proteins is considered to be the cause of numerous neurodegenerative conditions such as Alzheimer's disease [3]. Without doubt, there is a need to understand how and why proteins arrange themselves into oligomers. 
no. 290974)) for financial support. HMS thanks the Czech Science Foundation (grant no. P208/12/ G016).

Competing interests: The authors have declared that no competing interests exist.
The molecular dynamics (MD) simulation technique has been applied quite extensively to study the oligomerization and aggregation of membrane proteins including GPCRs [4-11] (see also the extensive review by Periole [12]) as well as members of other membrane protein classes $[13,14]$. Quite a few studies have also focused on the lateral diffusion dynamics in protein-rich membranes [15-18]. The most ambitious MD simulations have studied the assembly of respiratory chain supercomplexes [19] and the complete influenza A virion, whose surface is crowded with proteins [20].

All of these studies have used the coarse-grained (CG) Martini model [21-23] or its relatives [24] to probe time and length scales that are beyond the reach of fully atomistic simulations. Especially the Martini model has become very popular in the biomolecular simulation community due to its large library of molecule types, efficiency, as well as availability of high-quality simulation and analysis tools [25-30]. Martini has been parametrized [21] to contain a fairly limited set of bead types, each describing a group of $2-4$ heavy atoms. This guarantees speed, simplicity, and transferability, while it also provides an adequate level of chemical specificity for many applications. Notably, the Lennard-Jones (LJ) interactions between the 18 bead types are described by a total of 9 interaction levels with the interaction strength $\epsilon$ ranging from 2.0 to $5.6 \mathrm{~kJ} / \mathrm{mol}$, and the interaction distance parameter $\sigma$ having either a value of 0.62 (super repulsive type) or $0.47 \mathrm{~nm}$ (all other types). Additional "small" beads used in rings are described with $\sigma=0.43 \mathrm{~nm}$ together with $\epsilon$ that is reduced by $25 \%$ of the normal values. The original Martini model [21] also contains a limited set of bonded parameters. The masses are set to either $72 \mathrm{amu}$ or $45 \mathrm{amu}$ ("small types"), and partial charges exist as multiples of $1 \mathrm{e}$.

The bead types used in the Martini model were selected to provide liquid state conditions at room temperature and also to reproduce the partitioning coefficients of selected solutes between water and organic solvents [21]. Similarly, the Martini protein model [22] was parametrized using the same bead types, though the originally limited bonded interactions were expanded to provide correct geometries for the different amino acids and protein secondary structures. The performance of the parametrization was assessed based on the partitioning of amino acids between water and cyclohexane [22]. The protein force field was later fine-tuned based on the partitioning free energy between water and decane [23]. Even though numerous other checks were performed at both stages of the parametrization-including the evaluation of some amino acid dimerization free energies- $[22,23]$ the strength of protein-protein interactions - which cannot be predicted from those of individual amino acids-was actually not validated against experimental data [31].

This issue was brought up recently as Stark et al. [32] showed that interactions between water-soluble proteins in the Martini model are pronounced, leading to precipitation at concentrations much below the solubility limit [23]. By using the second osmotic virial coefficient as the target experimental value, Stark et al. [32] showed that it can be reproduced if the Lennard-Jones interaction strength between protein beads is drastically weakened [32]. In the same spirit, dimerization free energies calculated for membrane proteins and peptides indicate a very strong tendency for dimerization $[11,12,33-38]$ as exemplified by the dimerization free energies of about $-150 \mathrm{~kJ} / \mathrm{mol}$ and $-160 \mathrm{~kJ} / \mathrm{mol}$ reported for outer membrane protein $\mathrm{F}$ $(\mathrm{OmpF})[38]$ and $\kappa$-opioid receptor (KOPr) [11], respectively. Even though free energies cannot be directly linked to dimerization kinetics, it is obvious that such strong affinities indicate irreversible binding.

Förster resonance energy transfer (FRET) enables the calculation of dimerization free energies in simple model liposomes [39] as well as in liposomes constructed from mammalian plasma membrane extracts [40]. FRET provides association constants, which can be converted to dimerization free energy values allowing direct comparison of simulation with experiment. Still, it must be kept in mind that the values provided by FRET are obtained indirectly and are 
certainly not free of uncertainties. Notably, a dimer is defined as a state in which the fluorescent labels attached to the monomers-residing outside the membrane-are reasonably close to each other. Despite these limitations, FRET can be considered to be the most reasonable technique to provide dimerization free energies of membrane proteins in lipid membranes.

Consistent experimental dimerization data exist for certain protein types, allowing a straightforward comparison to simulations. The transmembrane (TM) domains of proteins in the receptor tyrosine kinase (RTK) class are a prime example. To begin with, FRET reported a value of $-10.5 \mathrm{~kJ} / \mathrm{mol}$ for the TM segment of an ErbB1 receptor in a DLPC bilayer [41]. Meanwhile, a Martini simulation of this dimer in a DPPC bilayer provided a much larger value of $-25.5 \mathrm{~kJ} / \mathrm{mol}$ [42], and a recent study with a Martini-derived force field on a ErbB1 homology model in a DPPC bilayer reported a value of $-38 \pm 3 \mathrm{~kJ} / \mathrm{mol}$ [43]. The dimerization free energy of another RTK TM segment, the Ephrin type-A receptor 1 (EphA1), was measured by FRET to be $-15.4 \mathrm{~kJ} / \mathrm{mol}$ in a DMPC bilayer [44]. Meanwhile, Martini simulation provided a substantially larger value of $-60 \mathrm{~kJ} / \mathrm{mol}$ in a DPPC bilayer [34]. Even though simulation data do not exist for the fibroblast growth factor receptor 3 (FGRF3) that is also a member of the RTK class, experiments in POPC liposomes reported dimerization free energies between $-11.3 \mathrm{~kJ} / \mathrm{mol}$ and $-12.6 \mathrm{~kJ} / \mathrm{mol}$ even when mutations are present [45-48]. Concluding, a number of results for the RTK TM domains point to dimerization free energies in the ballpark of -10 to $-15 \mathrm{~kJ} / \mathrm{mol}$, while Martini simulations consistently provide too stable dimers with dimerization free energies ranging between -25 and $-60 \mathrm{~kJ} / \mathrm{mol}$. While there is still room for speculation since the computational setups do not usually match the experimental ones, the significant discrepancy between experiment and Martini model predictions gives rise to concern.

The disagreement is not always this evident. For example, FRET experiments reported Glycophorin A (GpA) dimerization free energies in the range of -(13-17) $\mathrm{kJ} / \mathrm{mol}$ in various cell membranes [40,49], while Martini simulations in model bilayers provided much larger values of $-26 \mathrm{~kJ} / \mathrm{mol},-35 \mathrm{~kJ} / \mathrm{mol}$, and $-40 \mathrm{~kJ} / \mathrm{mol}$ [50-52]. However, experiments performed using a steric trap obtained values of $-50.6 \mathrm{~kJ} / \mathrm{mol}$ for GpA in a POPC membrane [53], while values of $-31.6 \mathrm{~kJ} / \mathrm{mol}$ [54] and $-31.4 \mathrm{~kJ} / \mathrm{mol}$ [55] were measured by a GALLEX assay in E. coli inner membranes.

Similarly, the dimerization free energy of the WALP23 peptide was calculated from a Martini simulation in a DOPC membrane to be $-20 \mathrm{~kJ} / \mathrm{mol}$ [56], while values of $-12.2 \pm 2 \mathrm{~kJ} / \mathrm{mol}$ [57] and $-15 \mathrm{~kJ} / \mathrm{mol}$ [58] were measured in DOPC and DLiPC bilayers, respectively. These values can also be compared to those measured for the structurally similar (AALALAA) ${ }_{3}$ peptide, for which a value of $-12.7 \pm 0.4 \mathrm{~kJ} / \mathrm{mol}$ was obtained with FRET [59]. On the other hand, the dimerization free energy of these (AALALAA) ${ }_{3}$ peptides was seen to be well reproduced with Martini but not with an atomistic force field [31]. Interestingly, a study comparing Martini with several atomistic force fields indicates that the atomistic force fields usually display lower dimerization free energies and some even underestimate them in comparison to experiments. [31]

The large scatter of the above listed experimental values that depend on the experimental technique and setup complicates comparison to simulations. However, most of the listed evidence points towards Martini predicting too strong dimerization. This is a potentially severe issue, since too large dimerization free energies lead to several problems: 1 . Extracting information on the lateral diffusion in realistic crowded-like conditions is compromised due to pronounced aggregation, which unrealistically confines the diffusion of proteins as well as lipids. 2. Resolving favorable lipid-protein interactions in membranes with multiple proteins is undermined as the affinity of proteins for the lipid binding sites is higher than that of lipids. 3 . Studying protein-protein dimerization interfaces becomes incomplete even in exceedingly 
long Martini simulations as proteins aggregate upon first contact and do not properly sample all possible configurations.

In this work, we show that the dimerization free energies obtained using the non-polarizable and polarizable variants of Martini for TM segments of RTKs are substantially larger than those measured in FRET experiments. We also show that the agreement between the simulation-based structures of spontaneously formed TM segment dimers and the structures resolved using NMR leaves a lot of room for improvement. We attempt to improve the situation of the non-polarizable Martini model by downscaling the protein-protein interactions without affecting other carefully parametrized parts of the Martini model. We find that the modification suggested by Stark et al. [32] (downscaling of the protein-protein LJ $\epsilon$ parameter by $60 \%$ ) is too drastic to resolve the excessive binding issue found with membrane proteins. On the other hand, applying this modification to only parts of the protein residing in the water phase does not reduce the dimerization energies sufficiently. Meanwhile, when we apply a milder $(10 \%)$ and uniform reduction in all protein-protein interactions, we find the dimerization free energies of TM segments to closely match experimental data, allowing for studies on membrane dynamics and protein-lipid interactions. However, the simulation structures of spontaneously formed dimers of transmembrane peptides are not improved, which calls for a more careful amino acid specific adjustment of the interaction levels.

\section{Materials and methods}

\section{Umbrella sampling simulations}

We consider RTK TM domain dimerization as a representative case for studying protein-protein interaction. This choice is made for two reasons. First, a large amount of experimental data exists for this protein class. Many TM domain dimer structures have been resolved by NMR and hence this information can be exploited to validate that the most favorable dimerization interface is studied in the simulations. Second, despite their flexibility, the convergence is more likely with TM domains formed by single helices rather than with more complex proteins, such as those composed of multiple helices or beta barrels.

The EphA1 dimer (PDB [60] entry 2K1L [61]) was embedded [27] in a lipid bilayer comprised of 400 DLPC molecules (indistinguishable from DMPC in Martini). The system was hydrated by 12.5 water beads (corresponding to 50 water molecules) per lipid out of which $10 \%$ were presented by the antifreeze type water beads. A physiological salt concentration of $150 \mathrm{mM}$ of NaCl was included to the system (in addition to counter ions) to match the setup employed in experiments [44]. See Fig 1 for selected snapshots of the system.

In addition, a dimer of ErbB1 TM domains (PDB [60] entry 2M0B [62]) was embedded into an identical DLPC bilayer. The only difference to the EphA1 system described above, in addition to the protein, was that the $\mathrm{NaCl}$ concentration was higher at $500 \mathrm{mM}$ in order to match the experimental setup [41].

The systems were first simulated for $50 \mathrm{~ns}$ with restraints on the dimer, so that the membrane was able to adapt to the newly included dimer. The restraints were then released and the system was simulated for another $50 \mathrm{~ns}$. Next, using the lateral distance between the centers of mass (COMs) of the TM domains as the reaction coordinate, the peptides were pulled apart to generate initial structures for umbrella sampling windows. In these initial structures, the COM distance varied between 0.6 and $5.0 \mathrm{~nm}$ with a spacing of $0.2 \mathrm{~nm}$.

The non-polarizable Martini model version 2.2 was employed [23] in the simulations together with the suggested "New-RF" simulation parameter set. [63] More concretely, the reaction field electrostatics and Lennard-Jones potentials were shifted to zero at the cut-off distance of $1.1 \mathrm{~nm}$. A dielectric constant of 15 was employed up to the cut-off length, after which 

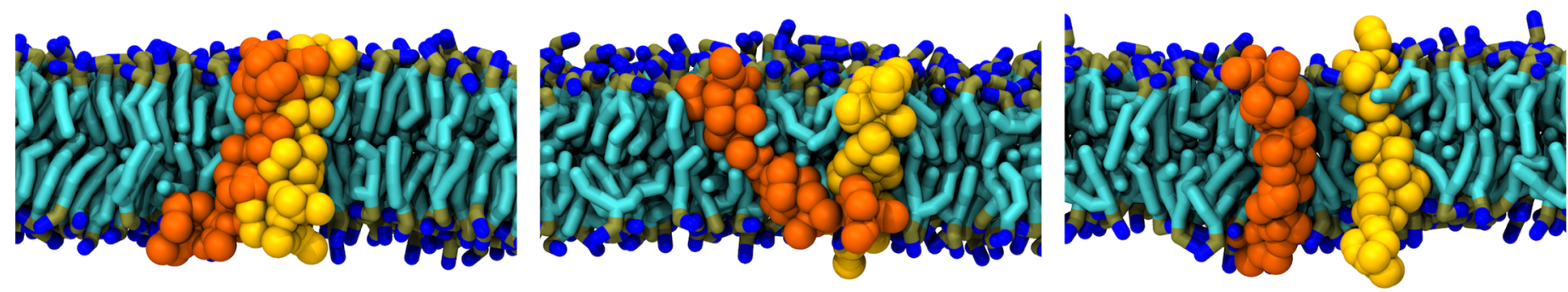

Fig 1. Selected snapshots of transmembrane domain dimerization. Examples of conformations at different COM-COM distances (from top to the bottom: 1.2, 2.0, and $2.4 \mathrm{~nm}$ ) from the umbrella sampling simulations (COM stands for the center of mass). Here, the U-0.1 scaling method is employed (see below). Chains of lipids (DLPC) are shown in cyan, phosphate beads in brown, and choline beads in blue. Peptides (EphA1) are shown in yellow and orange. Water, antifreeze particles, and ions have been omitted from the images.

it was given a value of infinity. The Verlet cut-off scheme was employed with parameters defined by Gromacs. Temperatures of the dimer, the lipids, and the solvent were separately kept constant at $303 \mathrm{~K}$ with the stochastic velocity rescaling thermostat [64], while pressure was maintained semi-isotropically at 1 bar using the Parrinello-Rahman barostat [65] with a time constant of $12 \mathrm{ps}$ and a compressibility of $3 \times 10^{-4} \mathrm{bar}^{-1}$. Additionally, as a reference we repeated the umbrella sampling simulations for the EphA1 dimer with the older set of suggested simulation parameters, referred to as "Common" (for details, see Ref. [63]), as well as using the recently updated polarizable parameters [66, 67], combined with the "New-RF" simulation parameters (with a dielectric constant of 2.5) [63]. In all simulations, the elastic network [68] with default options, i.e. a force constant of $500 \mathrm{~kJ} \mathrm{~mol}^{-1} \mathrm{~nm}^{-2}$ and an upper cut-off of $0.9 \mathrm{~nm}$ were employed for the TM domains.

For the umbrella sampling, the lateral distance of the COMs of the two TM segments was restrained with a harmonic spring with a constant of $400 \mathrm{~kJ} \mathrm{~mol}^{-1} \mathrm{~nm}^{-2}$, which provided a suitable overlap in the umbrella histograms. The selected umbrella windows (see Results) were each simulated for $10 \mu \mathrm{s}$ with a time step of $20 \mathrm{fs}$, and the first $50 \mathrm{~ns}$ of each window was discarded from analyses. The COM-COM distances were stored every 10 time steps (200 fs). Potential of mean force (PMF) profiles, estimating the free energy profile of dimerization $(G(r))$ were extracted using the g_wham tool [69]. The same tool was used to estimate the error bars in the PMF profiles using 100 bootstrap samples. All simulations were performed with the Gromacs version 5.0.x [70].

The dimerization free energy $\Delta G_{\text {DIM }}$ calculated from FRET experiments corresponds to the probability of the TM segments being in any bound state, i.e. the depth of the well in the free energy profile cannot be directly compared to experiment. Rather, an average needs to be calculated using [50]

$$
\Delta G_{\text {DIM }}=-R T \ln \left(K_{\mathrm{a}}\right)
$$

where

$$
K_{\mathrm{a}}=\pi \int_{0}^{r_{\max }} r \exp \left(-\frac{G(r)}{R T}\right) \mathrm{d} r
$$

Here, $K_{\mathrm{a}}$ is the association constant, $R$ the gas constant, $T$ the temperature, and $G(r)$ the free energy change (with respect to the chosen zero-level) at a COM-COM distance $r$. The usual standard state used in FRET experiments [40], $1 \mathrm{~nm}^{2}$ per receptor, is also adapted here. The $G(r)$ profiles are shifted to zero at a COM-COM distace of $2.75 \mathrm{~nm}$. Most profiles have reached a plateau at this arbitrarily chosen distance. The integration limit $\left(r_{\max }\right)$ is set to $2.0 \mathrm{~nm}$. The 
$r_{\max }$ value is larger than the used cutoff lengths, and the increase of $r_{\max }$ above $2.0 \mathrm{~nm}$ has an insignificant effect on the obtained $\Delta G_{\text {DIM }}$ value. The error in the $\Delta G_{\text {DIM }}$ values is the standard deviation of the individual values extracted from the 100 bootstrapped $G(r)$ samples.

\section{Spontaneous dimer formation}

We also evaluated the ability of the Martini model to predict the structures of TM domain dimers. For this purpose, we considered TM domains that are known to dimerize [71]. Notably, to this end we chose 1) Glycophorin A dimer (PDB entry 1AFO [72]), 2) integrin $\alpha \mathrm{IIb} \beta 3$ heterodimer (PDB entry 2K9J [73]), 3) BNIP3 TM domain dimer (PDB entry 2KA1 [74]), 4) T cell receptor signaling module $\zeta \zeta$ (PDB entry $2 \mathrm{HAC}$ [75]), and 5) DAP12 signaling subunit dimer (PDB entry 2L34 [76]). As initial simulation structures, the peptides in the dimer structures were separated by $\sim 4 \mathrm{~nm}$ and coarse-grained using the martinize tool, and embedded in a DLPC bilayer with insane [27]. The bilayers consisted of $\sim 400$ lipids and were hydrated by $\sim 6000$ water beads, out of which $10 \%$ was presented with the antifreeze type. About $150 \mathrm{mM}$ of $\mathrm{NaCl}$ was added to the water phase together with counter ions.

The assembled systems were first equilibrated for $50 \mathrm{~ns}$ with position restraints applied to the protein beads, after which $10 \mu$ s simulations for generating initial structures for the production simulations were performed for each system. During these simulations, the distance between the COMs of the peptides was kept at $\sim 4 \mathrm{~nm}$ with a harmonic potential with a spring constant of $400 \mathrm{~kJ} \mathrm{~mol}^{-1} \mathrm{~nm}^{-2}$. Next, a total of 10 structures were extracted at $1 \mu \mathrm{s}$ intervals, and employed as independent replicas with different initial structures. The 10 replica simulations were run with both unscaled Martini parameters as well as with the U-0.1 scaling strategy (see below). Therefore, the total number of these unbiased simulations was 5 (dimer structures) $\times 2$ (scaled vs. unscaled) $\times 10$ (replicas). Each of these simulations was run for $40 \mu$ s with the "New-RF" simulation parameters (see above), resulting in a total of $4 \mathrm{~ms}$ of simulation time.

The quality of the dimeric structures from these unbiased simulations was evaluated from the last $20 \mu$ s of simulations using three criteria: root-mean-square deviation (RMSD) of the backbone beads from the coarse-grained NMR structures of the dimer, the deviation of the dimer crossing angles from their values extracted from the coarse-grained NMR structures, and the deviations in the number of inter-peptide contacts (defined with a cut-off of $0.8 \mathrm{~nm}$ ) between the backbone beads in the coarse-grained NMR structure. The dimer crossing angles were calculated as the mean angles between the principal moments of inertia of the peptides calculated using a single value decomposition of the backbone beads of the residues defining the contact helices (See S1 Table in Supporting Information (SI)).

\section{Order of oligomerization}

We evaluated whether EphA1 and ErbB1 form dimers, as observed in experiments [41, 44], or higher order oligomers. To this end, we began with the structures extracted from the umbrella sampling windows with the lateral protein-protein distance equal to $\sim 3 \mathrm{~nm}$. We then replicated this system in the membrane plane four-fold $(2 \times 2)$. The systems containing a total of 8 monomers were simulated using both unscaled Martini parameters and the U-0.1 scaling strategy (see below) for $40 \mu$ s. The "New-RF" simulation parameters were again used (see above). The center of mass trajectories of the peptides were created with g_traj and analyzed with the tool g_clustsize. We used a cut-off of $2.5 \mathrm{~nm}$ for the clusters, as the radial distribution functions for the peptide COMs converged to zero at approximately this distance. 


\section{Adjustment of protein-protein interaction levels}

We consider two ways to scale down the LJ interactions among proteins in order to lower the dimerization free energies to a level observed in FRET experiments. These two approaches are described below. In Martini, the LJ interaction parameters are not calculated from combination rules but are instead tabulated as explained in the interaction matrix shown in the original Martini publication [21]. Therefore, the $\epsilon$ parameters considered here refer to the interaction parameters of two protein beads $\left(i, j\right.$, often denoted $\left.\epsilon_{i, j}\right)$ instead of the LJ parameter of a single bead.

Approach U: Uniform scaling applied to all beads. The Lennard-Jones interactions between all protein beads are here reduced by scaling down $\epsilon$ with a fixed scaling parameter $\alpha$ ( $\alpha=0.1$ for a reduction of $10 \%$, etc.). This method is identical (despite the reversed definition of $\alpha$ ) to that presented by Stark et al. [32] The scaled $\epsilon$-parameter $\epsilon_{\text {scaled }}$ calculated from the original value $\epsilon_{\text {original }}$ is

$$
\epsilon_{\text {scaled }}=\epsilon_{\text {repulsive }}+(1-\alpha)\left(\epsilon_{\text {original }}-\epsilon_{\text {repulsive }}\right),
$$

where $\epsilon_{\text {repulsive }}$ corresponds to the weakest (repulsive) interaction in the Martini model (2 kJ/ mol). Notably, in this method the values of $\epsilon$ are never smaller than $2 \mathrm{~kJ} / \mathrm{mol}$, which leads to a smaller spread of $\epsilon$ values and hence to less chemical specificity upon increasing $\alpha$. Unlike in the original work in the aqueous phase, we scale down the protein-protein interactions involving all bead types, including P4, Qa, and Qd. In this paper, the scaled results using this method will be labeled " $U-\alpha$ " with " $U$ " standing for uniform scaling.

Approach W: Scaling applied to water-contacting beads. Motivated by the good agreement with experiment based on scaling protein-protein interactions in water-soluble proteins by $60 \%(\alpha=0.6)$ [32], in this approach we scale down the protein-protein interactions of only those beads in membrane proteins that are mostly in contact with water (see details below). The interactions among these beads are adjusted following the approach " $U$ " described above. The results obtained using this method will be labeled "W- $\alpha$ " with "W" standing for water.

The beads to which the scaling was applied were determined as follows: First, the dimer was simulated for $1 \mu \mathrm{s}$, and the contacts between each residue with water and lipids were counted after a 50 ns equilibration period. Second, the sesidues that had more contacts with water than with lipids (defined by a cut-off of $0.6 \mathrm{~nm}$ ) were assigned new particle types, whose mutual interactions were scaled as in the " $U$ " approach. The interactions between scaled and unscaled protein beads remained as in standard Martini.

\section{Results and discussion}

\section{Dimerization free energies suggested by Martini are excessive}

Using the EphA1 and ErbB1 dimer systems, we evaluated the ability of the Martini model to capture the dimerization free energies of TM domains. Fig 2 depicts the free energy profiles (PMFs) calculated for the EphA1 and the ErbB1 dimer using either the non-polarizable ("NonPolar.") or the polarizable ("Pol.") Martini model. The use of "Common" simulation parameters instead of the "New-RF" ones is indicated by an asterisk $\left(^{*}\right)$. All curves have been shifted to realize convergence to zero at a COM-COM distance of $2.75 \mathrm{~nm}$. The values extracted from these profiles using Eqs (2) and (1) are shown in Table 1.

Using FRET, Artemenko et al. [44] measured a dimerization free energy of $\Delta G_{\mathrm{DIM}}=$ $-15.4 \pm 0.5 \mathrm{~kJ} / \mathrm{mol}$ for the EphA1 TM domains in DMPC liposomes at $303 \mathrm{~K}$ [44], i.e. in a setup matching our computational one. They also verified that EphA1 TM segments form dimers and not higher order oligomers. On the other hand, earlier Martini simulations of 


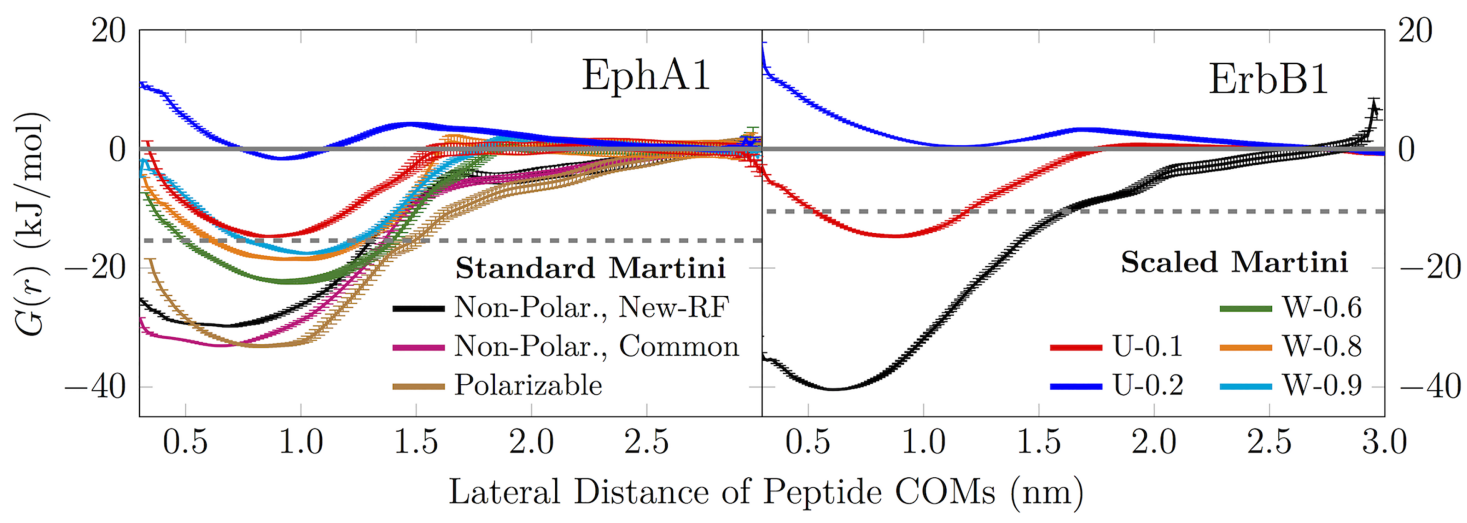

Fig 2. Dimerization free energy profiles of transmembrane domains. Free energy profiles of EphA1 and ErbB1 TM domain dimerization using either unmodified Martini or the "U" and "W" scaling strategies. The solid gray line at a value of zero is to guide the eye. The dashed gray line shows the experimental value for $\Delta G_{\mathrm{DIM}}$. Note that the common legend is split between the two panels.

https://doi.org/10.1371/journal.pone.0187936.g002

EphA1 in a DPPC membrane at $323 \mathrm{~K}$ provided a drastically different value of $-60 \pm 2 \mathrm{~kJ} / \mathrm{mol}$ for the depth of the well in the free energy profile [34].

As shown in Table 1, both the non-polarizable and polarizable Martini models provide values that are drastically higher than the ones obtained from experiments. The non-polarizable Martini provides a value of $-29.9 \pm 1.0 \mathrm{~kJ} / \mathrm{mol}$ that is approximately twice the experimental value. Importantly, the choice of simulation parameters ("New-RF" vs. "Common") has a small effect on the value: the dimerization free energy is slightly larger with the latter parameter set likely due to the longer cutoffs for non-bonded interactions. In addition, the polarizable Martini force field also provides very similar results suggesting that it also suffers from protein-protein overbinding. In all these simulations with an unscaled Martini model (non-polarizable or polarizable), the free energy profiles calculated do not fully reach a plateau even at an

Table 1. Transmembrane domain dimerization free energies.

\begin{tabular}{l|c|c} 
& EphA1 & ErbB1 \\
\hline Experimental $[41,44]$ & $-15.4 \pm 0.5$ & $-10.5 \pm 0.4$ \\
\hline Non-Polar. Martini & $-29.9 \pm 1.0$ & $-39.5 \pm 1.0$ \\
\hline Non-Polar. Martini* & $-32.9 \pm 0.5$ & - \\
\hline Polar. Martini & $-33.5 \pm 1.0$ & - \\
\hline Scaled U-0.1 & $-15.2 \pm 1.0$ & $-15.3 \pm 0.3$ \\
\hline Scaled U-0.2 & NA & NA \\
\hline Scaled W-0.6 & $-23.2 \pm 0.8$ & - \\
\hline Scaled W-0.8 & $-19.6 \pm 1.4$ & - \\
\hline Scaled W-0.9 & $-18.4 \pm 0.4$ & $-25.5 \pm 0.6$ \\
\hline Prev. Studies [34, 42, 43] & $-60 \pm 2$ & $-38 \pm 3$ \\
\hline
\end{tabular}

EphA1 and ErbB1 TM domain dimerization free energies $\Delta G_{\text {DIM }}$ (in $\mathrm{kJ} / \mathrm{mol}$ ) calculated from Eqs (2) and (1). In two cases, the dimer does not represent the minimum free energy state and hence $\Delta G_{\text {DIM }}$ values cannot be extracted (NA (not available)). The systems marked with "-" were not simulated. The system simulated with the "Common" parameter set instead of the "New-RF" (see Ref. [63]) setup is denoted with *. Standard and polarizable versions of Martini are denoted by "Non-Polar." and "Polar.", respectively.

https://doi.org/10.1371/journal.pone.0187936.t001 
inter-protein distance of $3 \mathrm{~nm}$. The protein-protein interactions are so strong that the monomers tilt to maintain any interaction at such large distances, resulting in a monotonous profile.

As to the ErbB1 dimer, FRET measurements by Chen et al. [41] in DLPC liposomes (with 500 $\mathrm{mM} \mathrm{NaCl}$ ) for $\Delta G_{\text {DIM }}$ of the TM domains provided $-10.5 \pm 0.5 \mathrm{~kJ} / \mathrm{mol}$. In these experiments, it was again verified that no higher order oligomers were formed. Notably, two independent FRET techniques were employed, and both of them provided almost identical $\Delta G_{\text {DIM }}$ values. A recent metadynamics study using the non-polarizable Martini model, on the other hand, provided a much larger value of $\Delta G_{\text {DIM }}=-38 \pm 3 \mathrm{~kJ} / \mathrm{mol}$ in a DPPC bilayer [43]. This value was obtained by a thorough sampling of the two-dimensional free energy surface (COM-COM separation and crossing angle). Additionally, an earlier Monte Carlo study using the Martini model in a DPPC bilayer at $323 \mathrm{~K}$ provided a value of $\Delta G_{\mathrm{DIM}}=-25.5 \pm 0.6 \mathrm{~kJ} / \mathrm{mol}$ [42].

The value reported by Lelimousin et al. [43] $\left(\Delta G_{\text {DIM }}=-38 \pm 3 \mathrm{~kJ} / \mathrm{mol}\right)$ is in excellent agreement with the value we obtained here with the standard Martini model $\left(\Delta G_{\text {DIM }}=-39.5 \pm 1.0\right.$ $\mathrm{kJ} / \mathrm{mol}$ ). This suggests that the two-dimensional sampling technique is likely not necessary when the structure of the dimer is readily available from experiments, as is the case with the two TM domains used as examples in this work.

Similarly as for the EphA1 dimer, our free energy profiles converge at unusually large interprotein distances indicating that the monomers try to remain in contact by tilting toward each other. Indeed, as shown in S1 Fig for the ErbB1 dimer using the non-polarizable Martini (see the SI), the peptides show a systematic tilting at large inter-protein distances.

The experiments for EphA1 and ErbB1 dimerization [41, 44] suggested that neither of these peptides forms higher order oligomers. Using unscaled Martini, we observed that EphA1 rapidly forms two tetramers, while ErbB1 oligomerizes slower but eventually assembles into an octamer. The time evolution of the average size of the oligomers is shown on the left in S3 Fig, and the histogram of the oligomer sizes during the last $10 \mu$ s of the simulation on the right in S3 Fig.

\section{Martini cannot predict dimer structures correctly}

We also evaluated the ability of the non-polarizable Martini model to predict the structures of five dimers of TM domains, resolved by NMR (see Methods). This provides an alternate comparison between simulation and experiment to the free energies presented in the previous section.

The COM-COM distance of the two peptides is shown in S2 Fig (see the SI), while a careful structural comparison between spontaneously formed dimers and the NMR structures is summarized in Fig 3.

The distances shown in S2 Fig (see the SI) indicate that all observed TM domains form dimers in a few microseconds, as expected. Fig 3 shows that simulations with the standard (non-polarizable) Martini are not able to predict the structures of spontaneously formed dimers correctly. In this figure, the simulation structure coinciding perfectly with the NMR structure would be a purple dot located at the origin. While it is difficult to decide how large deviations from the perfect match are acceptable, we have chosen to highlight in green (Fig 3) a deviation of $10^{\circ}$ (crossing angle) and $1 \mathrm{~nm}$ (RMSD) that we consider to be reasonable. This choice should help in assessing how well the simulation results agree with experimental data. Importantly, our data clearly show that the number of inter-peptide contacts in the dimer is often severely overestimated for the standard Martini, in line with the excessive dimerization free energies. Interestingly, for the 2L34 dimer, all replicas using the standard Martini fall in the same point in the graph, while other dimers show larger scatter of the replicas indicating a more diverse set of molecular conformations. This might result from the irreversible dimerization of the peptides upon first contact. 


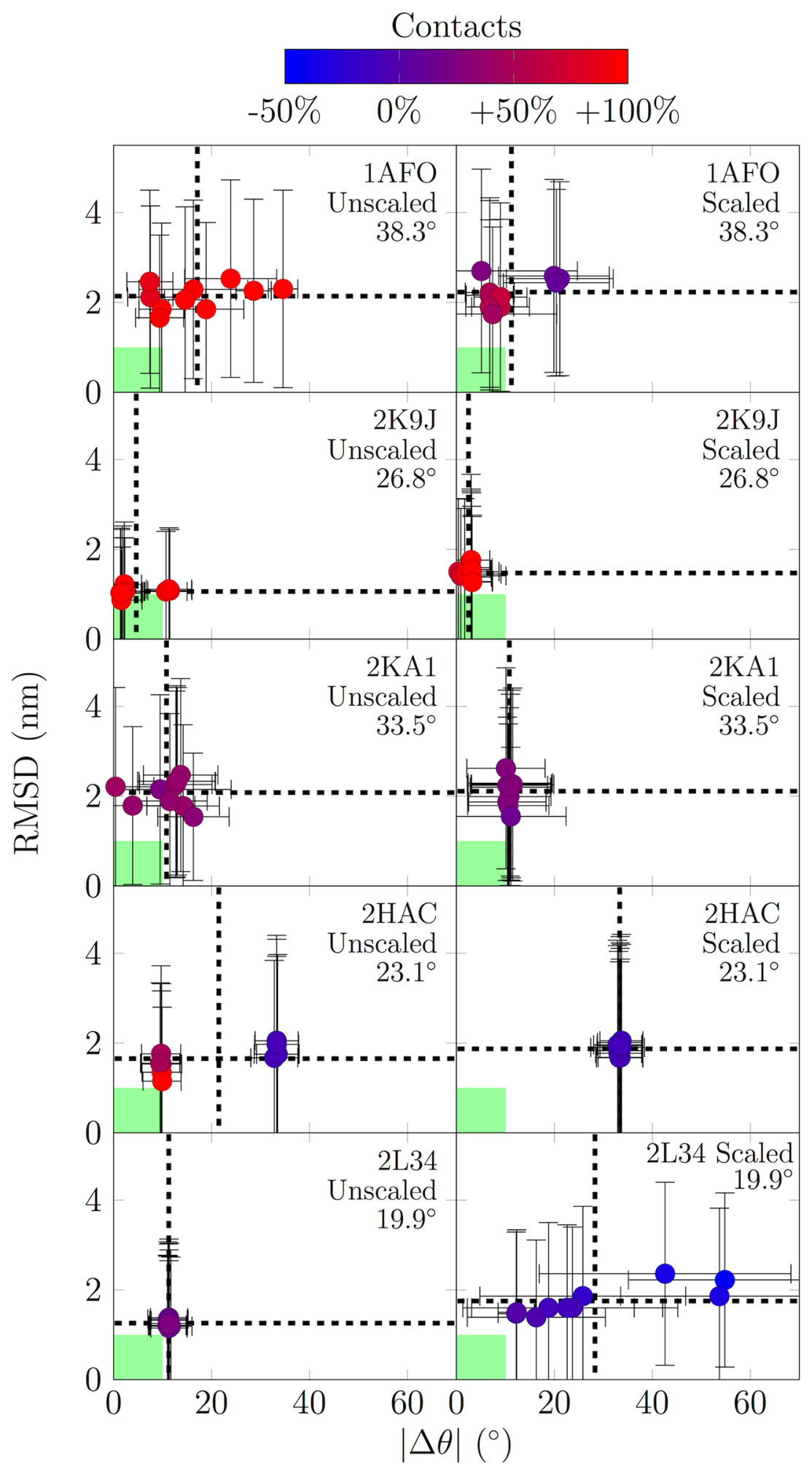

Fig 3. Deviation of the spontaneously formed dimer structures from their NMR structures. Data is shown for both the standard (non-polarizable) Martini and the U-0.1 scaling. The root-mean-square deviation (RMSD) is shown on the $y$ axis, and the absolute value of the deviation of the dimer crossing angle on the $x$ axis. The coloring shows the deviation in the number of backbone bead contacts (cutoff of $0.8 \mathrm{~nm}$ ). Therefore, an optimal result would be a purple dot (correct number of contacts) at the origin (RMSD equal to $0 \mathrm{~nm}$ and 
with the correct dimer crossing angle). As a rule of thumb, to guide the eye, the acceptable region (deviations up to $10^{\circ}$ and $1 \mathrm{~nm}$ considered to be acceptable) is highlighted in green. In each graph, data are shown for 10 replica simulations. Each replica is depicted with a marker with error bars showing standard deviation, while the mean over replicas is highlighted by the dashed lines. The crossing angle of the NMR structure is given for each dimer in degrees. Data are extracted from the last $20 \mu \mathrm{s}$ of the simulations.

https://doi.org/10.1371/journal.pone.0187936.g003

Still our approach of projecting the dimer structures onto three collective variables does not provide a full picture of the quality of the structures. To provide a more intuitive comparison between simulation and experiment, the final structures of all replicas of the spontaneous dimerization simulations are shown together with the corresponding NMR structures in Fig 4. Here, the structures are aligned to the NMR structure based on an RMSD fit of the dimerization motif of the first (in PDB) peptide, shown in yellow [71]. This motif is highlighted by purple spheres and the obtained fitted replicas are depicted in gray. The other (not RMSDaligned) peptide from NMR is shown in green. Finally, the position of the not RMSD-aligned peptides from the replicas is shown with colors from a spectrum of red-white-blue. This representation allows a straightforward evaluation of the spontaneously formed dimers by simply comparing the location of the second peptide observed in simulations (red-white-blue) with its location in the NMR structure (green).

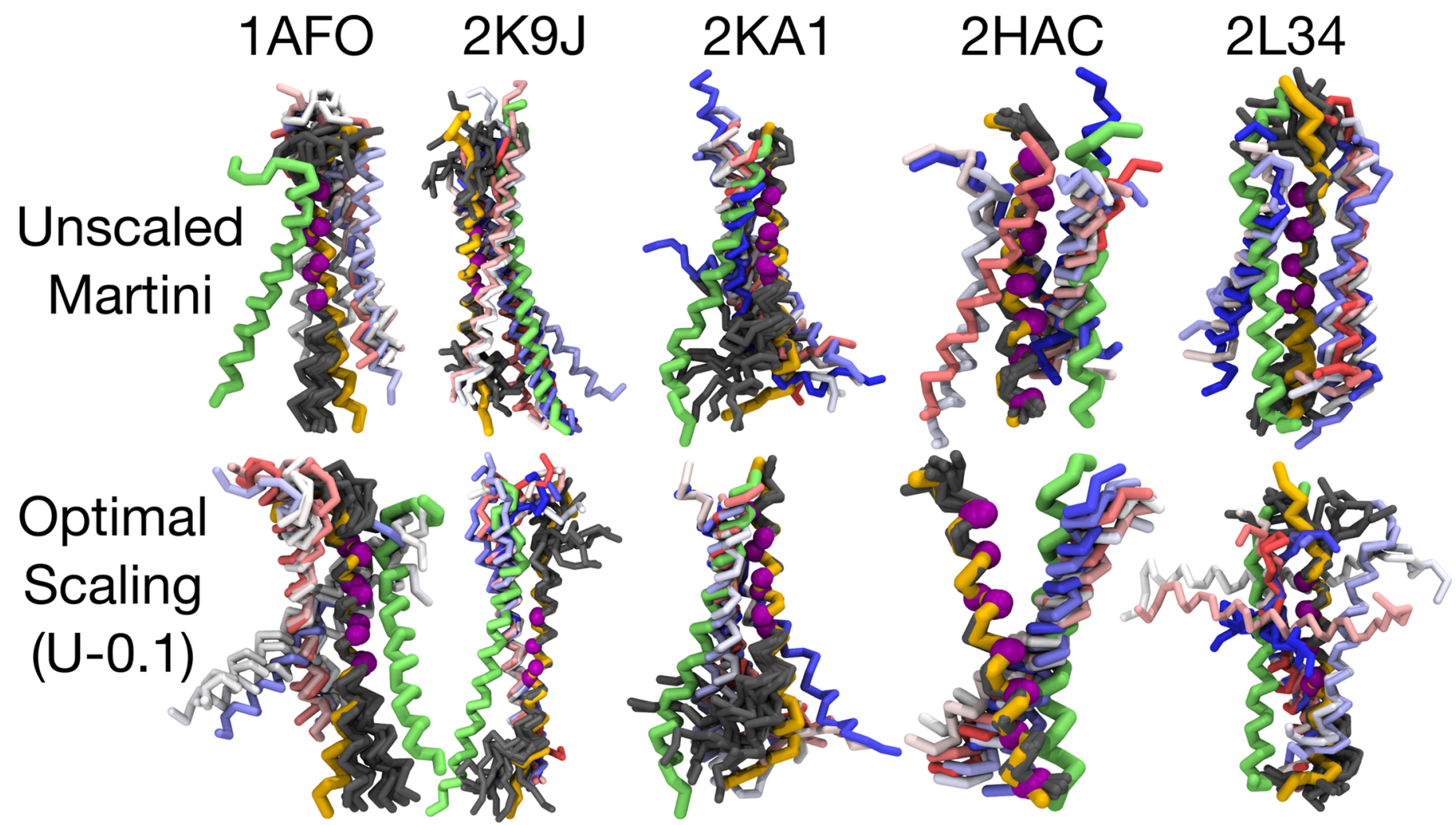

Fig 4. Final structures of the 10 validation replica simulations. Shown here are the structures for both standard (non-polarizable) Martini and the best scaling strategy resolved in this work (U-0.1). The two peptides in the NMR structure are shown in yellow (first peptide) and green (second peptide). The dimerization motif (see Ref. [71]) of the first peptide is additionally highlighted in purple. The dimer structures based on the simulations are depicted in a manner, where the first peptides of the replicas (all shown in gray) are RMSD-aligned at the dimerization motif (purple) on the first peptide of the NMR structure (yellow). The reader should here concentrate on the second peptide structures (not aligned) given by the 10 replica simulations that are depicted by a spectrum of red-white-blue. If there is good agreement between simulation and NMR, the structures of the second peptide (red-white-blue) should coincide with the second peptide in the NMR structure (green). 
It is evident that in most cases the simulations are unable to predict the experimentally resolved dimer conformation, supporting the poor agreement between simulation and experiment suggested by Fig 3 . The standard Martini often predicts conformations in which peptides are parallel to each other to maximize the number of inter-peptide contacts. In cases like 1AFO, the dimers form at the wrong interface. This is evident from the positioning of the second peptide in the NMR structures (shown in green) on the other side of the first peptide (shown in yellow) for all the replicas (shown in red-white-blue). For 2K9J, there is some overlap with the simulation replicas and the NMR structure. However, this agreement does not occur at the dimerization site. Instead, the peptides are almost parallel, resulting in too many inter-peptide contacts (see also Fig 3). For 2KA1, there is again some overlap at a correct site. However, for 2HAC, Martini shows multiple conformations indicating irreversible binding upon first contact. Finally, for 2L34, the conformations are again mutually inconsistent among the replicas.

While these results are not very encouraging, we must point out that the dimerization free energies calculated earlier are not affected by the poor prediction of dimer configurations, as the dimer configurations were obtained directly from structures resolved with NMR. It must be kept in mind, though, that the crossing angle deviation, RMSD, and the deviation in the number of inter-peptide backbone contacts were compared to the NMR structure, which is not always determined in a bilayer environment.

\section{Performance of the scaling approaches}

Having demonstrated the poor agreement between the standard Martini and experimental results, we move on to evaluate whether the issues can be corrected by scaling down the protein-protein interactions. As a first test, for the EphA1 dimer, we attempted the "W-0.6" fix, i.e. scaling down the interactions among the beads in contact with water. This approach was based on the promising results obtained by Stark et al. on water-soluble proteins [32]. This approach provided a value of $-23.2 \pm 0.8 \mathrm{~kJ} / \mathrm{mol}$, which is $\sim 50 \%$ too high compared to experimental results. Even further reduction of the interactions among the water-touching residues (W-0.8 or W-0.9) does not reduce the value of $\Delta G_{\mathrm{DIM}}$ to the experimental value (see Table 1), providing concrete evidence that also the hydrophobic residues need to be scaled. Even if this strong scaling had provided good agreement with the experiment, it would have removed all chemical specificity from the model, since for example at $\alpha=0.9$ the most repulsive and the most attractive interactions are separated by only $\sim 0.4 \mathrm{~kJ} / \mathrm{mol}(2.0$ vs. $2.36 \mathrm{~kJ} / \mathrm{mol}$ ).

Next, we applied the uniform scaling approach ("U") to the EphA1 dimer. We first scaled the $L J \epsilon$ values using scaling factors $\alpha$ in the range of $0.1-0.9$ with a spacing of 0.1 (see Eq (3)). After $250 \mathrm{~ns}$ of free energy (umbrella sampling) simulations per window, the obviously wrong values of $\alpha$ in each strategy were discarded, and the simulations were continued to $10 \mu$ s per window using only the most promising candidates (U-0.1 and $\mathrm{U}-0.2)$. Also, the number of simulated windows was limited at this point, as only the windows that were required to reach the convergence of free energy profiles were extended to $10 \mu$ s. Depending on the scaling approach and the dimer, this plateau was reached at $2.6-3.6 \mathrm{~nm}$.

As shown in Table 1, the best agreement with experiment with the EphA1 dimer is obtained with the U-0.1 approach, i.e. when the protein-protein interactions among all protein beads are reduced by $10 \%$. This approach gives a value of $-15.2 \pm 1.0 \mathrm{~kJ} / \mathrm{mol}$, which is consistent, within error margins, with the value obtained from experiment. Using stronger scaling ( $\alpha>$ $0.1)$ leads to unstable dimerization as the minimum in the free energy profile vanishes.

Applying the U-0.1 approach to the ErbB1 dimer, we obtained a value of $-15.3 \pm 0.3 \mathrm{~kJ} /$ mol, i.e. the dimer is somewhat too stable in the simulation but the dimerization free energy is 
still sufficiently low for reversible binding that allows sampling of different dimerization interfaces (see Table 1). Meanwhile, stronger scaling did not lead to a stable dimer. This hints that a uniform approach is not sufficient, but a more careful amino-acid specific adjustment of the protein-protein interactions might be required.

Next, we verified that the U-0.1 scaling approach is gentle enough to not result in the unfolding of proteins. The S1 File (see the SI) demonstrates that protein structures are stable also with the scaling.

Having established U- 0.1 as the most promising scaling approach, we evaluated its performance in predicting the structures of the spontaneously forming dimers as well as the level of oligomerization. These are tasks for which the standard Martini was shown above to be not optimal. As shown in S3 Fig, using the U-0.1 scaling, the eight ErbB1 monomers form one tetramer and two dimers, which is a clear improvement over the octamer observed for standard Martini. For EphA1, on the other hand, the performance was not improved by the U-0.1 scaling, and two tetramers were observed. It is not possible to ensure that the clustering process has reached equilibrium in our simulations. However, it is unlikely that the formed oligomers would decrease in size if the simulations were extended further. Equilibrium oligomer sizes are overestimated for both models. However, we observe several promising unbinding events with the scaled model, which suggests that the scaling takes the behavior of the system in the correct direction.

Moving on to the structures of spontaneously formed dimers, as shown in S2 Fig, the dimers form spontaneously even when the protein-protein interactions are reduced, however the formation of dimers takes more time when the U-0.1 scaling is employed. This allows the dimers to sample dimerization interfaces before a stable dimer is formed. Even one brief dissociation event is observed for the $2 \mathrm{~K} 9 \mathrm{~J}$ dimer with the $\mathrm{U}-0.1$ scaling.

Unfortunately, as shown in Fig 4, the agreement with experiment is not improved systematically with the U-0.1 scaling, and as with the standard Martini, all replicas fall outside the region highlighted in green. The scaled Martini model provides mutually consistent behavior among the replicas for most of the dimers (2K9J, $2 \mathrm{KA} 1$, and $2 \mathrm{HAC}$ ). However, the only decent agreement with experiment is observed for $2 \mathrm{HAC}$, for which all replicas show substantial overlap with the NMR structure. However, the deviations grow towards the bottom part of the structure resulting in a substantial deviation in the tilt angle (see Fig 3).

At this point, it is clear that the properties discussed here seem to be very sensitive to the scaling parameters. As an example, free energies of dimerization can change by multiple dozen kilojoules per mole upon a decrease of only $10 \%$ in the Lennard-Jones interactions. Similarly, this small decrease affects the structures of dimers quite drastically as shown in Fig 3. Based on these observations, any scaling scheme should be always adapted with care, and its validity should be evaluated by comparison with experiment.

The U-0.1 scaling should benefit studies on membrane dynamics as it prevents excessive and unrealistic clustering. It should also help proteins sample protein dimerization interfaces before actually forming a dimer. One would also assume that a uniform scaling would not affect the preference of the protein dimerization interfaces. However, the proper sampling of dimerization interfaces of membrane proteins such as GPCRs requires substantial effort and simulation time $[7,28,35,43]$. Since such a thorough study is beyond the scope of this work, the validity of the scaling approach in predicting protein dimerization interfaces remains to be studied.

With the apparent limitations in the ability of the standard Martini model to describe protein-protein interactions, and considering that our two approaches to adjust the interaction levels also fail in predicting the correct dimerization interfaces, the obvious question is how the situation could be improved. Our results show that the reduction in protein-protein 
interactions required for membrane proteins to reproduce experimental dimerization free energies $(10 \%)$ is much smaller than for water-soluble proteins (60\% [32]). Importantly, the amino acid content in these two kinds of proteins is very different, suggesting that an amino acid specific scaling strategy, even though being very laborious and beyond the scope of this work, could provide an approach applicable to a wide range of protein types. Notably, such a technique would be especially crucial for studies involving full-length membrane proteins where ectodomains and cytosolic regions are largely exposed to interactions with water, while the TM domains are mainly in a hydrophobic environment. Currently, however, such amino acid specific scaling is also limited by the lack of quantitative experimental data for validation, covering both membrane proteins and water soluble proteins, let alone proteins with segments inside and outside the membrane.

Membrane lipids also play a key role in the oligomerization of membrane proteins. A recent study by Castillo et. al showed that a large hydrophobic mismatch can stabilize a dimer substantially [56]. Furthermore, the role of membrane lipids in altering the rate and the interface of receptor oligomerization were also recently demonstrated by computer simulations [7, 77]. In the free energy calculations described in this work, the role of lipids was accounted for; the membrane compositions followed those used in the respective experiments, and the correct dimerization interfaces were sampled as the conformations were derived from the dimer structure resolved by NMR. Also, the changes in $\Delta G_{\text {DIM }}$ due to hydrophobic mismatch [56] were smaller than the observed deviations between experiments and Martini and cannot therefore explain their poor mutual agreement. Furthermore, in our recent work [7] we observed that scaling down protein-protein interactions decreased the rate of oligomerization systematically for two different lipid compositions pointing out to a general trend that cannot be explained by the choice of lipids.

Coarse-grained simulations lack many details provided by their atomistic counterparts. Notably, in addition to van der Waals forces and steric repulsion, entropic effects and hydrogen bonding are also captured in the LJ potential in the coarse-grained scheme. Therefore, in order to resolve the nature of protein-protein and protein-lipid interactions in detail, the finegraining of the simulated structures to atomistic resolution, followed by further simulation, is recommended. Fortunately, the recent developments in resolution transformation tools (martinize and backward [29]) provide easy access to such effective multiscale simulations. In these simulations, it is of crucial importance that the interactions are reasonably similar at the used resolutions. Otherwise efficient CG simulations might take the system to an unfavorable state-such as a tightly bound protein aggregate or a protein with its lipid binding sites covered by other proteins-from which the united-atom or all-atom simulations cannot escape.

\section{Conclusions}

Membrane protein dimerization free energies calculated using the coarse-grained Martini model [21-23] are often much larger than the corresponding experimental values by tens of kilojoules per mole. If dimerization free energies in simulation models are too large, or even excessive, they can result in instantaneous, nonselective, and irreversible binding, thereby leading to formation of unrealistic protein oligomers or superaggregates. This further complicates the interpretation of simulation data for dimerization interfaces: if the binding between two proteins is disproportionately strong and fast, then the dimerization interface predicted by coarse-grained simulations may be wrong. Also, the aggregates resulting from excessively large dimerization free energies may give rise to unrealistically pronounced confinement effects for lipids and proteins diffusing in the membrane plane, thereby impairing the lateral dynamics of 
membrane systems. All these issues complicate the interpretation of experimental data with the aid of molecular dynamics simulations based on the coarse-grained Martini force field.

In this work, we attempted to improve this situation by scaling the interaction between the membrane proteins. The results based on this strategy are partly encouraging. We found that only a small reduction of approximately $10 \%$ in the $\mathrm{LJ}$ interactions is required to bring the dimerization free energies of RTK TM domains to the same ballpark with values obtained from FRET experiments. This signals that the Martini parametrization together with its simple combination rules gets impressively close to optimal values in a task that the Martini model was never parametrized for. Meanwhile, the small reduction in the LJ interactions did not improve agreement between the structures of spontaneously formed dimers found in simulations and those resolved from NMR, and the degree of oligomerization was still too large as compared to experiments.

Martini has also occasionally been able to predict the most favorable dimer structures [35, 43], even though their absolute binding energies are not in line with experiment. In this case, the slight and universal decrease of protein-protein interactions will likely not affect the relative affinities of the interfaces, yet it will allow the proteins to sample more conformations until they find the optimal orientation. However, this remains to be confirmed in future studies.

To conclude, our study brings about an issue in the description of protein-protein interactions in the Martini model. We consider that our work sets a promising basis for further studies to design a more accurate parametrization strategy based on, e.g., amino acid specific scaling of protein-protein interactions. In the meantime, the scaled version of Martini, as discussed in this paper, can be used to improve studies on membrane dynamics and proteinlipid interactions. However, in all cases a careful comparison of simulation predictions with biochemical experiments must be performed whenever possible.

\section{Supporting information}

S1 Table. Dimer reference crossing angles. Residues used for the calculation of dimeric crossing angles. Pept- 1 and Pept-2 show the two peptides consituting the dimers. The indicated residues from both peptides (numbering starting from 1) were used in the calculation; the crossing angle is defined as the angle between the principal moments of inertia of the backbone beads of the indicated residues. The angle extracted from the PDB structures is indicated by "Ang-PDB", while that calculated in this work is labeled "Ang-Exp.". Note that we omit the handedness and only show positive crossing angle values.

(XLSX)

S1 Fig. Monomer tilt angles. Tilt angles of ErpB1 monomers with respect to the membrane normal as a function of COM-COM separation (COM stands for the centre of mass). The systematic increase of the angle, seen in the unscaled Martini model (red and black curves), is due to the strong protein-protein interaction: when the peptides are separated, they tilt in order to keep the termini at one end in contact with each other. (PDF)

S2 Fig. Peptide-peptide distances. Distance between the peptide COMs as a function of simulation time in the unbiased simulations. Data are shown for the 10 repeats done for each system, and the insets show the data for the last $20 \mu$ s in more detail. (PDF)

S3 Fig. Order of oligomerization. A) The time evolution of the average oligomer size of EphA1 and ErbB1 peptides. B) The histogram of the oligomer sizes during the last $10 \mu$ s of the 
simulation.

(PDF)

S1 File. Stability of BtuB membrane protein with the U-0.1 scaling. Details of a simulation of a BtuB membrane protein. Stability analysis comparing the results between the normal (unscaled) Martini model and the U-0.1 scaling. (PDF)

\section{Acknowledgments}

We acknowledge Leija Lahtinen for her help in the early stages of the project. CSC-IT Center for Science (Espoo, Finland) is acknowledged for computing resources.

\section{Author Contributions}

Conceptualization: Matti Javanainen, Hector Martinez-Seara, Ilpo Vattulainen.

Data curation: Matti Javanainen, Hector Martinez-Seara.

Formal analysis: Matti Javanainen, Hector Martinez-Seara.

Funding acquisition: Hector Martinez-Seara, Ilpo Vattulainen.

Investigation: Matti Javanainen, Hector Martinez-Seara.

Methodology: Matti Javanainen, Hector Martinez-Seara.

Project administration: Hector Martinez-Seara, Ilpo Vattulainen.

Resources: Matti Javanainen, Ilpo Vattulainen.

Supervision: Hector Martinez-Seara, Ilpo Vattulainen.

Validation: Matti Javanainen, Hector Martinez-Seara.

Visualization: Matti Javanainen.

Writing - original draft: Matti Javanainen, Hector Martinez-Seara.

Writing - review \& editing: Matti Javanainen, Hector Martinez-Seara, Ilpo Vattulainen.

\section{References}

1. Ferré S, Casadó V, Devi LA, Filizola M, Jockers R, Lohse MJ, et al. G Protein-Coupled Receptor Oligomerization Revisited: Functional and Pharmacological Perspectives. Pharmacol Rev. 2014; 66(2):413434. https://doi.org/10.1124/pr.113.008052 PMID: 24515647

2. Gomes I, Ayoub MA, Fujita W, Jaeger WC, Pfleger KD, Devi LA. G Protein-Coupled Receptor Heteromers. Annu Rev Pharmacol Toxicol. 2016; 56:403-425. https://doi.org/10.1146/annurev-pharmtox011613-135952 PMID: 26514203

3. Haass C, Selkoe DJ. Soluble Protein Oligomers in Neurodegeneration: Lessons From the Alzheimer's Amyloid $\beta$-Peptide. Nat Rev Mol Cell Biol. 2007; 8(2):101-112. https://doi.org/10.1038/nrm2101 PMID: 17245412

4. Ghosh A, Sonavane U, Joshi R. Multiscale Modelling to Understand the Self-Assembly Mechanism of Human $\beta 2$-Adrenergic Receptor in Lipid Bilayer. Computat Biol Chem. 2014; 48:29-39. https://doi.org/ 10.1016/j.compbiolchem.2013.11.002

5. Mondal S, Johnston JM, Wang H, Khelashvili G, Filizola M, Weinstein H. Membrane Driven Spatial Organization of GPCRs. Sci Rep. 2013; 3:2909. https://doi.org/10.1038/srep02909 PMID: 24105260

6. Periole X, Huber T, Marrink SJ, Sakmar TP. G Protein-Coupled Receptors Self-Assemble in Dynamics Simulations of Model Bilayers. J Am Chem Soc. 2007; 129(33):10126-10132. https://doi.org/10.1021/ ja0706246 PMID: 17658882 
7. Guixà-González R, Javanainen M, Gómez-Soler M, Cordobilla B, Domingo JC, Sanz F, et al. Membrane Omega-3 Fatty Acids Modulate the Oligomerisation Kinetics of Adenosine $A_{2 A}$ and Dopamine $D_{2}$ Receptors. Sci Rep. 2016; 6. https://doi.org/10.1038/srep19839 PMID: 26796668

8. Provasi D, Boz MB, Johnston JM, Filizola M. Preferred Supramolecular Organization and Dimer Interfaces of Opioid Receptors From Simulated Self-Association. PLoS Comput Biol. 2015; 11(3): e1004148. https://doi.org/10.1371/journal.pcbi.1004148 PMID: 25822938

9. Koldsø H, Sansom MS. Organization and Dynamics of Receptor Proteins in a Plasma Membrane. J Am Chem Soc. 2015; 137(46):14694-14704. https://doi.org/10.1021/jacs.5b08048 PMID: 26517394

10. Johnston JM, Aburi M, Provasi D, Bortolato A, Urizar E, Lambert NA, et al. Making Structural Sense of Dimerization Interfaces of Delta Opioid Receptor Homodimers. Biochemistry. 2011; 50(10):1682-1690. https://doi.org/10.1021/bi101474v PMID: 21261298

11. Johnston JM, Filizola M. Differential Stability of the Crystallographic Interfaces of Mu-And Kappa-Opioid Receptors. PLoS One. 2014; 9(2):e90694. https://doi.org/10.1371/journal.pone.0090694 PMID: 24651466

12. Periole X. Interplay of G Protein-Coupled Receptors With the Membrane: Insights From Supra-Atomic Coarse Grain Molecular Dynamics Simulations. Chem Rev. 2016; 117(1):156-185. https://doi.org/10. 1021/acs.chemrev.6b00344 PMID: 28073248

13. Parton DL, Klingelhoefer JW, Sansom MS. Aggregation of Model Membrane Proteins, Modulated by Hydrophobic Mismatch, Membrane Curvature, and Protein Class. Biophys J. 2011; 101(3):691-699. https://doi.org/10.1016/j.bpj.2011.06.048 PMID: 21806937

14. Rassam P, Copeland NA, Birkholz O, Tóth C, Chavent M, Duncan AL, et al. Supramolecular Assemblies Underpin Turnover of Outer Membrane Proteins in Bacteria. Nature. 2015; 523(7560):333-336. https://doi.org/10.1038/nature14461 PMID: 26061769

15. Javanainen $\mathrm{M}$, Hammaren $\mathrm{H}$, Monticelli L, Jeon JH, Miettinen MS, Martinez-Seara $\mathrm{H}$, et al. Anomalous and Normal Diffusion of Proteins and Lipids in Crowded Lipid Membranes. Faraday Disc. 2013; 161:397-417. https://doi.org/10.1039/C2FD20085F

16. Jeon JH, Javanainen $M$, Martinez-Seara H, Metzler R, Vattulainen I. Protein Crowding in Lipid Bilayers Gives Rise to Non-Gaussian Anomalous Lateral Diffusion of Phospholipids and Proteins. Phys Rev X. 2016; 6(2):021006.

17. Goose JE, Sansom MS. Reduced Lateral Mobility of Lipids and Proteins in Crowded Membranes. PLoS Comput Biol. 2013; 9(4):e1003033. https://doi.org/10.1371/journal.pcbi.1003033 PMID: 23592975

18. Chavent M, Reddy T, Goose J, Dahl ACE, Stone JE, Jobard B, et al. Methodologies for the Analysis of Instantaneous Lipid Diffusion in MD Simulations of Large Membrane Systems. Faraday Disc. 2014; 169:455-475. https://doi.org/10.1039/C3FD00145H

19. Arnarez C, Marrink S, Periole X. Molecular Mechanism of Cardiolipin-Mediated Assembly of Respiratory Chain Supercomplexes. Chem Sci. 2016; 7:4435-4443. https://doi.org/10.1039/C5SC04664E

20. Reddy T, Shorthouse D, Parton DL, Jefferys E, Fowler PW, Chavent M, et al. Nothing to Sneeze At: A Dynamic and Integrative Computational Model of an Influenza a Virion. Structure. 2015; 23(3):584-597. https://doi.org/10.1016/j.str.2014.12.019 PMID: 25703376

21. Marrink SJ, Risselada HJ, Yefimov S, Tieleman DP, De Vries AH. The MARTINI Force Field: Coarse Grained Model for Biomolecular Simulations. J Phys Chem B. 2007; 111(27):7812-7824. https://doi. org/10.1021/jp071097f PMID: 17569554

22. Monticelli L, Kandasamy SK, Periole X, Larson RG, Tieleman DP, Marrink SJ. The MARTINI CoarseGrained Force Field: Extension to Proteins. J Chem Theory Comput. 2008; 4(5):819-834. https://doi. org/10.1021/ct700324x PMID: 26621095

23. de Jong DH, Singh G, Bennett WD, Arnarez C, Wassenaar TA, Schäfer LV, et al. Improved Parameters for the Martini Coarse-Grained Protein Force Field. J Chem Theory Comput. 2012; 9(1):687-697. https://doi.org/10.1021/ct300646g PMID: 26589065

24. Bond PJ, Sansom MS. Insertion and Assembly of Membrane Proteins via Simulation. J Am Chem Soc. 2006; 128(8):2697-2704. https://doi.org/10.1021/ja0569104 PMID: 16492056

25. Stansfeld PJ, Goose JE, Caffrey M, Carpenter EP, Parker JL, Newstead S, et al. MemProtMD: Automated Insertion of Membrane Protein Structures Into Explicit Lipid Membranes. Structure. 2015; 23 (7):1350-1361. https://doi.org/10.1016/j.str.2015.05.006 PMID: 26073602

26. Qi Y, Ingólfsson HI, Cheng X, Lee J, Marrink SJ, Im W. CHARMM-GUI Martini Maker for CoarseGrained Simulations With the Martini Force Field. J Chem Theory Comput. 2015; 11(9):4486-4494. https://doi.org/10.1021/acs.jctc.5b00513 PMID: 26575938

27. Wassenaar TA, Ingólfsson HI, Böckmann RA, Tieleman DP, Marrink SJ. Computational Lipidomics With insane: A Versatile Tool for Generating Custom Membranes for Molecular Simulations. J Chem Theory Comput. 2015; 11(5):2144-2155. https://doi.org/10.1021/acs.jctc.5b00209 PMID: 26574417 
28. Wassenaar TA, Pluhackova K, Moussatova A, Sengupta D, Marrink SJ, Tieleman DP, et al. HighThroughput Simulations of Dimer and Trimer Assembly of Membrane Proteins. The DAFT Approach. J Chem Theory Comput. 2015; 11(5):2278-2291. https://doi.org/10.1021/ct5010092 PMID: 26574426

29. Wassenaar TA, Pluhackova K, Böckmann RA, Marrink SJ, Tieleman DP. Going Backward: A Flexible Geometric Approach to Reverse Transformation From Coarse Grained to Atomistic Models. J Chem Theory Comput. 2014; 10(2):676-690. https://doi.org/10.1021/ct400617g PMID: 26580045

30. Javanainen M, Martinez-Seara H. Efficient Preparation and Analysis of Membrane and Membrane Protein Systems. Biochim Biophys Acta. 2016; 1858(10):2468-2482. https://doi.org/10.1016/j.bbamem. 2016.02.036 PMID: 26947184

31. Nishizawa M, Nishizawa K. Potential of Mean Force Analysis of the Self-Association of Leucine-Rich Transmembrane $\alpha$-Helices: Difference Between Atomistic and Coarse-Grained Simulations. J Chem Phys. 2014; 141(7):075101. https://doi.org/10.1063/1.4891932 PMID: 25149815

32. Stark AC, Andrews CT, Elcock AH. Toward Optimized Potential Functions for Protein-Protein Interactions in Aqueous Solutions: Osmotic Second Virial Coefficient Calculations Using the Martini CoarseGrained Force Field. J Chem Theory Comput. 2013; 9(9):4176-4185. https://doi.org/10.1021/ ct400008p

33. Dunton TA, Goose JE, Gavaghan DJ, Sansom MS, Osborne JM. The Free Energy Landscape of Dimerization of a Membrane Protein, NanC. PLoS Comput Biol. 2014; 10(1):e1003417. https://doi.org/ 10.1371/journal.pcbi.1003417 PMID: 24415929

34. Chavent M, Chetwynd AP, Stansfeld PJ, Sansom MS. Dimerization of the EphA1 Receptor Tyrosine Kinase Transmembrane Domain: Insights Into the Mechanism of Receptor Activation. Biochemistry. 2014; 53(42):6641-6652. https://doi.org/10.1021/bi500800x PMID: 25286141

35. Periole X, Knepp AM, Sakmar TP, Marrink SJ, Huber T. Structural Determinants of the Supramolecular Organization of G Protein-Coupled Receptors in Bilayers. J Am Chem Soc. 2012; 134(26):1095910965. https://doi.org/10.1021/ja303286e PMID: 22679925

36. Johnston JM, Wang H, Provasi D, Filizola M. Assessing the Relative Stability of Dimer Interfaces in G Protein-Coupled Receptors. PLoS Comput Biol. 2012; 8(8):e1002649. https://doi.org/10.1371/journal. pcbi.1002649 PMID: 22916005

37. Provasi D, Johnston JM, Filizola M. Lessons From Free Energy Simulations of $\delta$-Opioid Receptor Homodimers Involving the Fourth Transmembrane Helix. Biochemistry. 2010; 49(31):6771-6776. https://doi.org/10.1021/bi100686t PMID: 20617813

38. Casuso I, Khao J, Chami M, Paul-Gilloteaux P, Husain M, Duneau JP, et al. Characterization of the Motion of Membrane Proteins Using High-Speed Atomic Force Microscopy. Nat Nanotech. 2012; 7 (8):525-529. https://doi.org/10.1038/nnano.2012.109

39. You M, Li E, Wimley WC, Hristova K. Förster Resonance Energy Transfer in Liposomes: Measurements of Transmembrane Helix Dimerization in the Native Bilayer Environment. Anal Biochem. 2005; 340(1):154-164. https://doi.org/10.1016/j.ab.2005.01.035 PMID: 15802141

40. Chen L, Novicky L, Merzlyakov M, Hristov T, Hristova K. Measuring the Energetics of Membrane Protein Dimerization in Mammalian Membranes. J Am Chem Soc. 2010; 132(10):3628-3635. https://doi. org/10.1021/ja910692u PMID: 20158179

41. Chen L, Merzlyakov M, Cohen T, Shai Y, Hristova K. Energetics of ErbB1 Transmembrane Domain Dimerization in Lipid Bilayers. Biophys J. 2009; 96(11):4622-4630. https://doi.org/10.1016/j.bpj.2009. 03.004 PMID: 19486684

42. Prakash A, Janosi L, Doxastakis M. Self-Association of Models of Transmembrane Domains of ErbB Receptors in a Lipid Bilayer. Biophys J. 2010; 99(11):3657-3665. https://doi.org/10.1016/j.bpj.2010.10. 023 PMID: 21112290

43. Lelimousin M, Limongelli V, Sansom MS. Conformational Changes in the Epidermal Growth Factor Receptor: Role of the Transmembrane Domain Investigated by Coarse-Grained Metadynamics Free Energy Calculations. J Am Chem Soc. 2016; 138:10611-10622. https://doi.org/10.1021/jacs.6b05602 PMID: 27459426

44. Artemenko EO, Egorova NS, Arseniev AS, Feofanov AV. Transmembrane Domain of EphA1 Receptor Forms Dimers in Membrane-Like Environment. Biochim Biophys Acta. 2008; 1778(10):2361-2367. https://doi.org/10.1016/j.bbamem.2008.06.003 PMID: 18590698

45. Li E, You M, Hristova K. FGFR3 Dimer Stabilization Due to a Single Amino Acid Pathogenic Mutation. J Mol Biol. 2006; 356(3):600-612. https://doi.org/10.1016/j.jmb.2005.11.077 PMID: 16384584

46. Li E, You M, Hristova K. Sodium Dodecyl Sulfate-Polyacrylamide Gel Electrophoresis and Förster Resonance Energy Transfer Suggest Weak Interactions Between Fibroblast Growth Factor Receptor 3 (FGFR3) Transmembrane Domains in the Absence of Extracellular Domains and Ligands. Biochemistry. 2005; 44(1):352-360. https://doi.org/10.1021/bi048480k PMID: 15628877 
47. Merzlyakov M, Chen L, Hristova K. Studies of Receptor Tyrosine Kinase Transmembrane Domain Interactions: The EmEx-FRET Method. J Membr Biol. 2007; 215(2-3):93-103. https://doi.org/10.1007/ s00232-007-9009-0 PMID: 17565424

48. You M, Li E, Hristova K. The Achondroplasia Mutation Does Not Alter the Dimerization Energetics of the Fibroblast Growth Factor Receptor 3 Transmembrane Domain. Biochemistry. 2006; 45(17):55515556. https://doi.org/10.1021/bi060113g PMID: 16634636

49. Sarabipour S, Hristova K. Glycophorin a Transmembrane Domain Dimerization in Plasma Membrane Vesicles Derived From CHO, HEK 293T, and A431 Cells. Biochim Biophys Acta. 2013; 1828(8):18291833. https://doi.org/10.1016/j.bbamem.2013.03.022 PMID: 23562404

50. Sengupta D, Marrink SJ. Lipid-Mediated Interactions Tune the Association of Glycophorin a Helix and Its Disruptive Mutants in Membranes. Phys Chem Chem Phys. 2010; 12(40):12987-12996. https://doi. org/10.1039/c0cp00101e PMID: 20733990

51. Janosi L, Prakash A, Doxastakis M. Lipid-Modulated Sequence-Specific Association of Glycophorin a in Membranes. Biophys J. 2010; 99(1):284-292. https://doi.org/10.1016/j.bpj.2010.04.005 PMID: 20655857

52. Domański J, Hedger G, Best RB, Stansfeld PJ, Sansom MS. Convergence and Sampling in Determining Free Energy Landscapes for Membrane Protein Association. J Phys Chem B. 2017; 121(15):33643375. https://doi.org/10.1021/acs.jpcb.6b08445 PMID: 27807980

53. Hong H, Blois TM, Cao Z, Bowie JU. Method to Measure Strong Protein-Protein Interactions in Lipid Bilayers Using a Steric Trap. Proc Natl Acad Sci USA. 2010; 107(46):19802-19807. https://doi.org/10. 1073/pnas.1010348107 PMID: 21041662

54. Nash A, Notman R, Dixon AM. De Novo Design of Transmembrane Helix-Helix Interactions and Measurement of Stability in a Biological Membrane. Biochim Biophys Acta. 2015; 1848(5):1248-1257. https://doi.org/10.1016/j.bbamem.2015.02.020 PMID: 25732028

55. Finger C, Volkmer T, Prodöhl A, Otzen DE, Engelman DM, Schneider D. The Stability of Transmembrane Helix Interactions Measured in a Biological Membrane. J Mol Biol. 2006; 358(5):1221-1228. https://doi.org/10.1016/j.jmb.2006.02.065 PMID: 16574146

56. Castillo N, Monticelli L, Barnoud J, Tieleman DP. Free Energy of WALP23 Dimer Association in DMPC, DPPC, and DOPC Bilayers. Chem Phys Lipids. 2013; 169:95-105. https://doi.org/10.1016/j. chemphyslip.2013.02.001 PMID: 23415670

57. Schäfer LV, de Jong DH, Holt A, Rzepiela AJ, de Vries AH, Poolman B, et al. Lipid Packing Drives the Segregation of Transmembrane Helices Into Disordered Lipid Domains in Model Membranes. Proc Natl Acad Sci USA. 2011; 108(4):1343-1348. https://doi.org/10.1073/pnas.1009362108 PMID: 21205902

58. Ash WL. Helix-Helix Interactions in Membrane Proteins Probed With Computer Simulations. University of Calgary; 2009.

59. Yano Y, Matsuzaki K. Measurement of Thermodynamic Parameters for Hydrophobic Mismatch 1: SelfAssociation of a Transmembrane Helix. Biochemistry. 2006; 45(10):3370-3378. https://doi.org/10. 1021/bi052286w PMID: 16519531

60. Berman HM, Westbrook J, Feng Z, Gilliland G, Bhat TN, Weissig H, et al. The Protein Data Bank. Nucl Acids Res. 2000; 28(1):235-242. https://doi.org/10.1093/nar/28.1.235 PMID: 10592235

61. Bocharov EV, Mayzel ML, Volynsky PE, Goncharuk MV, Ermolyuk YS, Schulga AA, et al. Spatial Structure and $\mathrm{pH}$-Dependent Conformational Diversity of Dimeric Transmembrane Domain of the Receptor Tyrosine Kinase EphA1. J Biol Chem. 2008; 283(43):29385-29395. https://doi.org/10.1074/jbc. M803089200 PMID: 18728013

62. Bocharov EV, Lesovoy DM, Pavlov KV, Pustovalova YE, Bocharova OV, Arseniev AS. Alternative Packing of EGFR Transmembrane Domain Suggests That Protein-Lipid Interactions Underlie Signal Conduction Across Membrane. Biochim Biophys Acta. 2016; 1858(6):1254-1261. https://doi.org/10. 1016/j.bbamem.2016.02.023 PMID: 26903218

63. de Jong DH, Baoukina S, Ingólfsson HI, Marrink SJ. Martini Straight: Boosting Performance Using a Shorter Cutoff and GPUs. Comput Phys Commun. 2016; 199:1-7. https://doi.org/10.1016/j.cpc.2015. 09.014

64. Bussi G, Donadio D, Parrinello M. Canonical Sampling Through Velocity Rescaling. J Chem Phys. 2007; 126(1):014101. https://doi.org/10.1063/1.2408420 PMID: 17212484

65. Parrinello M, Rahman A. Polymorphic Transitions in Single Crystals: A New Molecular Dynamics Method. J Appl Phys. 1981; 52(12):7182-7190. https://doi.org/10.1063/1.328693

66. Yesylevskyy SO, Schäfer LV, Sengupta D, Marrink SJ. Polarizable Water Model for the Coarse-Grained MARTINI Force Field. PLoS Comput Biol. 2010; 6(6):e1000810. https://doi.org/10.1371/journal.pcbi. 1000810 PMID: 20548957 
67. Michalowsky J, Schäfer LV, Holm C, Smiatek J. A Refined Polarizable Water Model for the CoarseGrained MARTINI Force Field With Long-Range Electrostatic Interactions. J Chem Phys. 2017; 146 (5):054501. https://doi.org/10.1063/1.4974833 PMID: 28178817

68. Periole X, Cavalli M, Marrink SJ, Ceruso MA. Combining an Elastic Network With a Coarse-Grained Molecular Force Field: Structure, Dynamics, and Intermolecular Recognition. J Chem Theory Comput. 2009; 5(9):2531-2543. https://doi.org/10.1021/ct9002114 PMID: 26616630

69. Hub JS, De Groot BL, Van Der Spoel D. g_wham—a Free Weighted Histogram Analysis Implementation Including Robust Error and Autocorrelation Estimates. J Chem Theory Comput. 2010; 6(12):37133720. https://doi.org/10.1021/ct100494z

70. Abraham MJ, Murtola T, Schulz R, Páll S, Smith JC, Hess B, et al. GROMACS: High Performance Molecular Simulations Through Multi-Level Parallelism From Laptops to Supercomputers. SoftwareX. 2015; 1:19-25. https://doi.org/10.1016/j.softx.2015.06.001

71. Cymer F, Veerappan A, Schneider D. Transmembrane Helix-Helix Interactions Are Modulated by the Sequence Context and by Lipid Bilayer Properties. Biochim Biophys Acta. 2012; 1818(4):963-973. https://doi.org/10.1016/j.bbamem.2011.07.035 PMID: 21827736

72. MacKenzie KR, Prestegard JH, Engelman DM. A Transmembrane Helix Dimer: Structure and Implications. Science. 1997; 276(5309):131-133. https://doi.org/10.1126/science.276.5309.131 PMID: 9082985

73. Lau TL, Kim C, Ginsberg MH, Ulmer TS. The Structure of the Integrin allb $\beta 3$ Transmembrane Complex Explains Integrin Transmembrane Signalling. EMBO J. 2009; 28(9):1351-1361. https://doi.org/10. 1038/emboj.2009.63 PMID: 19279667

74. Sulistijo ES, MacKenzie KR. Structural Basis for Dimerization of the BNIP3 Transmembrane Domain. Biochemistry. 2009; 48(23):5106-5120. https://doi.org/10.1021/bi802245u PMID: 19415897

75. Call ME, Schnell JR, Xu C, Lutz RA, Chou JJ, Wucherpfennig KW. The Structure of the $\zeta \zeta$ Transmembrane Dimer Reveals Features Essential for Its Assembly With the T Cell Receptor. Cell. 2006; 127 (2):355-368. https://doi.org/10.1016/j.cell.2006.08.044 PMID: 17055436

76. Call ME, Wucherpfennig KW, Chou JJ. The Structural Basis for Intramembrane Assembly of an Activating Immunoreceptor Complex. Nat Immunol. 2010; 11(11):1023-1029. https://doi.org/10.1038/ni.1943 PMID: 20890284

77. Prasanna X, Chattopadhyay A, Sengupta D. Cholesterol Modulates the Dimer Interface of the $\beta_{2^{-}}$ Adrenergic Receptor via Cholesterol Occupancy Sites. Biophys J. 2014; 106(6):1290-1300. https://doi. org/10.1016/j.bpj.2014.02.002 PMID: 24655504 